\title{
SNX10 and PTGDS are Associated with the Progression and Prognosis of Cervical Squamous Cell Carcinoma
}

\author{
Pinping Jiang \\ Nanjing Medical University \\ Ying Cao \\ Nanjing Medical University \\ Feng Gao \\ Nanjing Medical University \\ Wei Sun \\ Nanjing Medical University \\ Jinhui Liu \\ Nanjing Medical University

\section{Ziyan Ma}

University of New South Wales

\section{Manxin Xie}

Nanjing Medical University

Shilong Fu ( $\square$ docfusl@163.com )

Jiangsu Province Hospital and Nanjing Medical University First Affiliated Hospital https://orcid.org/0000-0001-7416-3070

\section{Research article}

Keywords: Cervical squamous cell carcinoma, methylation, prognosis, risk model, SNX10, PTGDS

Posted Date: January 12th, 2021

DOI: https://doi.org/10.21203/rs.3.rs-50778/v4

License: (1) (i) This work is licensed under a Creative Commons Attribution 4.0 International License.

Read Full License 


\section{Abstract}

Background: Cervical cancer (CC) is the primary cause of death in women. This study sought to investigate the potential mechanism and prognostic genes of CC.

Methods: We downloaded four gene expression profiles from GEO. The RRA method was used to integrate and screen differentially expressed genes (DEGs) between CC and normal samples. Functional analysis was performed by clusterprofiler. We built PPI network by Search Tool for the Retrieval of Interacting Genes Database (STRING) and selected hub modules via Molecular COmplex Detection (MCODE). CMap database was used to find molecules with therapeutic potential for CC. The hub genes were validated in GEO datasets, Gene Expession Profiling Interactive Analysis (GEPIA), immunohistochemistry, Cox regression analysis, TCGA methylation analysis and ONCOMINE were carried out. ROC curve analysis and GSEA were also performed to describe the prognostic significance of hub genes.

Results: Functional analysis revealed that 147 DEGs were significantly enriched in binding, cell proliferation, transcriptional activity and cell cycle regulation. PPI network screened 30 hub genes, with CDK1 having the strongest connectivity with CC. Cmap showed that apigenin, thioguanine and trichostatin A might be used to treat $C C(P<0.05)$. Eight genes (APOD, CXCL8, MMP1, MMP3, PLOD2, PTGDS, SNX10 and SPP1) were screened out through GEPIA. Of them, only PTGDS and SNX10 had not appeared in previous studies about CC. The validation in GEO showed that PTGDS showed low expression in tumor tissues while SNX10 showed high expression in tumor tissues. Their expression profiles were consistent with the results in immunohistochemistry. ROC curve analysis indicated that the model had a good diagnostic efficiency $(A U C=0.738)$. GSEA showed that the two genes were associated with the chemokine signaling pathway $(P<0.05)$. TCGA methylation analysis showed that patients with lowly-expressed and highly-methylated PTGDS had a worse prognosis than those with highly-expressed and lowly-methylated PTGDS ( $p=0.037)$. Cox regression analysis showed that SNX10 and PTGDS were independent prognostic indicators for OS among CC patients( $P=0.007$ and 0.003$)$.

Conclusions: PTGDS and SNX10 showed abnormal expression and methylation in CC. Both genes might have high prognostic value of CC patients.

\section{Background}

An annual death toll of 265,700 makes cervical cancer (CC) the second deadliest malignancy in females [1]. Despite pre-cancerous screening and emerging treatments, $\mathrm{CC}$ remains the primary cause of death in women in developing countries [2]. When CC metastasizes and recurs, the prognosis gets even worse. Therefore, it is of great significance to create new treatments of CC based on its to-be-clarified molecular mechanism.

Gene expression microarray, as an efficient means of acquiring large-scale genetic data, is being widely used to study gene expression profiling in many human cancers. Upon microarray and databases, 
effective analytic tools have been designed to explore tumor-associated genes, molecular mechanisms and target therapies. The integration of databases containing gene expression chips allows in-depth study of molecular mechanisms[3, 4].

Thousands of differentially expressed genes (DEGs) in CC have been discovered [5-7]. However, the results on some mRNAs are inconsistent. Here we use an unbiased approach to solve this problem.

In our study, we screened DEGs from four profiles downloaded from GEO. PPI network was built by STRING Database and hub modules selected via plug-in MCODE. CMap was used to find potential genes associated with CC. We also validated hub genes with GEO, GEPIA, immunohistochemistry and ONCOMINE. ROC curve analysis and GESA were also done to tease out the significance of hub genes. The flow chart of this research was displayed in Figure 1.

\section{Methods}

\subsection{Screening DEGs}

Keywords "cervical cancer geo accession" were put in the GEO database (https://www.ncbi.nlm.nih.gov/geo/) and the gene expression profiles of GSE6791, GSE63514, GSE39001 and GSE9750 were downloaded. The dataset information is shown in Table 1. We processed unqualified data by $\mathrm{R}$ package. The data is calibrated, standardized and log2-transformed. Gene expression analysis was performed using the limma[8] package in the Bioconductor package. Relevant codes were placed into R. We selected four microarray datasets and analyzed them with limma. The |log2fold change (FC)| > 2 and adjusted $p<0.05$ were set as cutoffs. RRA package was download (http://cran.r-project.org/) [9] and $\mathrm{R}$ was used for running the instruction code.

\subsection{Functional analysis based on DEGs}

The biological significance of DEGs was analyzed with DAVID (https://david.ncifcrf.gov/) database and clusterprofiler[10] (a package visualizing the biological profiles of genes). $P<0.05$ was considered to be statistically significant.

\subsection{PPI network integration}

Search Tool for the Retrieval of Interacting Genes Database (STRING)[11] (http://www.string-db.org/) was used to assess PPI complex between identified and predicted proteins. In addition, the plug-in MCODE [12] of Cytoscape was conducted to select and visualize hub modules in PPI complex.

\subsection{Identification of potential drugs}

CMap[13] is a computer simulation method for predicting the potential drug that may induce or reverse a biological state encoded by the gene expression signature. The different probe components commonly between $\mathrm{CC}$ and normal samples were screened out with CMap database and divided into the up- and 
down-regulated groups. An enrichment score representing similarity was calculated. The positive score illustrated that the drug could induce cancer in human; the negative score illustrated the drug function oppositely and had potential therapeutic value.

\subsection{Construction of a prognostic signature}

Univariate Cox proportional hazard regression analysis was performed based on DEGs. The genes associated with prognosis were defined using the cutoff value of $p<0.05$. Next, a multivariate Cox regression model was constructed with genes of $p<0.01$. Cox regression with $p<0.05$ was constructed to estimate the risk score of each patient on the expression of the DEGs. To further screen out prognosisrelated genes of $\mathrm{CC}$, we constructed a linear risk model. The prognostic score $=$

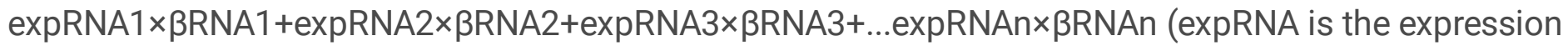
level of each methylation-driven gene, and $\beta R N A$ is the regression coefficient calculated by the multivariate Cox regression analysis). The prognostic risk value of each sample was calculated according to the formula, and then the median of the index value was cut off. Patients were divided into a low- and high-risk group according to their mean scores of prognostic risks. Kaplan-Meier survival analysis was conducted based on the low- and high-risk group. We also performed ROC curve analysis using five years as the predicted time to assess the predictive value of the outcome. The areas under the ROC curve, sensitivity and specificity were used to describe predictive values.

\subsection{Validation of key genes}

We used GEPIA[14] (Gene Expression Profiling Interactive Analysis) to analyze the expression and prognostic significance of DEGs. After reviewing literature, we screened real hub genes from DEGs. Subsequently the real hub genes were validated into GEO datasets, including GSE7803 and GSE29576, and ONCOMINE database (www.oncomine.org). GSE7803 included $21 \mathrm{CC}$ tissue samples and 10 normal tissue samples. GSE29576 included 45 CC tissue samples and 17 normal tissue samples. ONCOMINE[15] dataset is a publicly accessible online cancer microarray database that enables online analysis on relations between a candidate gene and various tumors according to DNA and RNA sequence data. The Human Protein Atlas (HPA) (http://www.proteinatlas.org/) was also used to validate the expression of the real hub genes. ROC curve analysis was performed to distinguish normal and cancer tissues.

\subsection{Survival analysis and mapping of methylation level}

Survival analysis on gene methylation and expression was conducted through $\mathrm{R}$ package to identify key prognosis-associated genes in CC. To explore the relation between aberrant methylation and expression of genes, we extracted key genes with methylated expression from the downloaded data on TCGA CESC methylation. Then we evaluated the association between the methylated expression and the gene expression.

\subsection{Gene set enrichment analysis (GSEA)}


Based on the hub gene expression level, the samples were divided into two groups. In order to study the potential function of the DEGs. GSEA[16] (http://software.broadinstitute.org/gsea/index.jsp) was used to research a series of prior defined biological pathways which might be enriched in the gene rank derived from hub gene between the two groups. Annotated gene set of c2.cp.kegg.v6.0.symbols.gmt in Molecular Signatures Database (MSigDB, http://software.broadinstitute.org/gsea/msigdb/index.jsp) was selected as the reference. Additionally, we used "Clusterprofiler" package in $\mathrm{R}$ to handle the datasets, and the "Enrichplot" package to tease out the enriched pathways of the key genes. The adjusted- $P<0.05$ was set as significant.

\section{Results}

\subsection{Identification of DEGs in CC}

The CC expression microarray datasets (GSE6791, GSE9750, GSE39001 and GSE63514) were firstly standardized (Figure S1). With limma package, 256 DEGs were filtered from GSE6791 (60 downregulated and 196 upregulated), 236 DEGs from GSE9750 (136 downregulated and 100 upregulated), 98 DEGs from GSE39001 (38 upregulated and 60 downregulated), 489 DEGs from GSE63514 (177 upregulated and 312 downregulated). DEGs from the 4 microarray datasets were exhibited in volcano maps (Figure S2A-D) and heatmaps (Figure S3A-D). We analyzed the four microarray datasets via the limma package and then with RRA method according to their log-folding variation values ( $(\mid \log 2$ fold change $(F C) \mid>1$ and adj. $p<0.05)$. The RRA method was based on the theory that genes in each experiment were randomly ordered. For the genes ranking higher in the experiment, the possibility of differential expression is inversely proportional to the value of $P$. Through analytic hierarchy analysis, we sorted out 74 upregulated genes, and 73 down-regulated genes (Table 2). Finally, the R-heatmap software was performed to plot the top 40 up- and down-regulated genes (Figure 2).

\subsection{Functional analysis of DEGs}

The biological annotations of DEGs in CC were obtained with an online analysis tool named DAVID, which had GO analysis of up- and down-regulated genes ( $P \otimes 0.05)$. The GO analysis of DEGs covered three aspects: molecular function, biological processes and cellular composition (Figure S4A). The upregulated genes were significantly enriched in microtubule binding, tubulin binding and ATPase activity (Figure 3A), and the down-regulated genes in serine-type peptidase activity, serine hydrolase activity and serine-type endopeptidase (Figure 3B). These results indicated that most DEGs were prominently enriched in structural molecule activity, midbody, kinesin complex and microtubule motor activity. (Figure S4 B-C and Figure S5A). Clusterprofile was performed to analyze the DEGs. The result showed that the upregulated genes were mostly enriched in DNA replication, Oocyte meiosis and Cell cycle. (Figure 3C), and the downregulated genes in Arachidonic acid metabolism, prostate cancer and signaling pathways regulating pluripotency of stem cells (Figure 3D). The pathway-gene network (Figure S5B) suggested that the cell cycle was the most important term in the biological processes of CC.

\subsection{PPI network construction and modules selection}


The PPI network of DEGs was constructed, including 147 nodes (74 up-regulated genes and 73 downregulated genes) and 562 edges (Figure 4A). Degrees $\geq 30$ was set as the cutoff. A total of 16 genes, such as CDK1, TOP2A, NCAPG, and KIF11, were showed the most significant difference in expression (Figure 4B). A significant module was selected with plug-in MCODE, including 27 nodes and 343 edges (Figure S6A). GO and KEGG pathway enrichment analysis indicated that the genes in this module were mainly related to microtubule binding, tubulin binding, cell cycle and oocyte mitosis (Figure S6B and 6C).

\subsection{Small molecule drugs screening}

CMap network was used to analyze 147 differentially expressed genes into two groups (74 in upregulated group and 73 in down-regulated group). After the signature query, the three compounds with the highest negative enrichment score (apigenin, thioguanine, and trichostatin A) were identified as potential therapeutic agents for CC (Table 3). The three-dimensional chemical structure of these three compounds is shown in Figure 5.

\subsection{Validation of hub genes}

We validated DEGs at GEPIA website, including survival analysis and tissue sample expression analysis (Figure S7 and Figure S8). Eight genes (APOD, CXCL8, MMP1, MMP3, PLOD2, PTGDS, SNX10 and SPP1) had the same trend in both the above analysis. We literature-reviewed these eight genes, finding that only PTGDS and SNX10 had not been reported to be associated with CC. Therefore, we used GSE7803 and GSE29576 to validate PTGDS and SNX10 (Figure S9). The results showed that PTGDS had high expression levels in normal tissues and low expression levels in tumor tissues, while SNX10 showed an opposite profile. We further validated the two genes using immunohistochemistry (Figure 6A-B) and ONCOMINE, obtaining the results consistent with those from the GEO database (Figure 6C-D). The area under the curve of PTGDS was 0.919 and that of PTGDS was 0.905 , suggesting that both can distinguish CC and normal tissue and have a good diagnostic efficiency (Figure 7A). GSEA was performed to search KEGG pathways enriched in the TCGA samples. The top ten most enriched pathways included "hematopoietic cell lineage", "adhesion molecules cams", "vascular smooth muscle contraction", "systemic lupus erythematosus", "chemokine signaling pathway", "t cell receptor signaling pathway", "cytokine cytokine receptor interaction", "calcium signaling pathway", "neuroactive ligand receptor interaction" and "leukocyte transendothelial migration" (Figure 7B) (adj. $p<0.05)$. In addition, the univariate and multivariate Cox proportional hazards regression analyses showed that SNX10 and PTGDS were independent prognostic indicators for OS among CESC patients ( $P=0.007$ and 0.003$)$ (Table 4).

To find out the mechanism of abnormal gene expression, we analyzed the gene expression level and methylation level from the Illumina Human Methylation 450 platform based on TCGA data. The association between the methylated expression and the gene expression of the two key driving genes were shown in Figure7C-D. The survival analysis showed that the patients with low-expressed and hypermethylated PTGDS had a worse prognosis than those with high-expressed and hypo-methylated 
PTGDS(P=0.037) (Figure 7E). However, SNX10 methylation has no statistical significance in survival analysis.

\subsection{Establishment of Cox regression model}

Univariate cox regression analysis screened out seven genes, including APOD, CXCL8, MMP1, MMP3, PLOD2, PTGDS and SPP1 (Figure S10). Multivariate Cox regression analysis screened five genes, including SPP1, CXCL8, PTGDS, PLOD2 and MMP3 (Figure S11). The score for predicting overall survival risk was calculated as followed: Risk score $=0.143 *$ SPP1+0.136* CXCL8-0.093* PTGDS+0.206* PLOD2+0.067* MMP3. Based on the risk score, CC patients were divided into low- and high-risk groups. Kaplan-Meier survival analysis suggested that low-risk patients had better overall survival than high-risk patients in the TCGA cohort (Figure 8A). ROC curve analysis was also completed according to the 5-year survival of the area under the receiver operating characteristic curve (AUC) value. The specificity and sensitivity were both highest when the risk score was 0.738 (Figure 8B). The distribution of risk score, survival status, and the expression levels of five genes was also analyzed (Figure $\mathbf{8 C}-\mathbf{F}$ ). The expression levels of five genes in low- and high-risk groups were shown in Figure S12A. The univariate and multivariate Cox proportional hazards regression analyses showed that only the risk score based on five genes was independent prognostic indictor of CC (Figure S12B-C).

The Heatmap showed the expression levels of the five genes in high- and low-risk patients in the TCGA dataset. We observed significant difference in survival state $(P<0.001)$ and stage $(P<0.05)$ (Figure $\mathbf{S}$ 12D).

\section{Discussion}

CC brings on more than 265,700 deaths per year, making it the second deadlist malignancy in women. Nowadays, microarray and high-throughput sequencing technology are used to identify the potential targets in CC treatment. Previous studies often establish a single group or have a small-size, which restricts their reliability.

This study analyzed the expression profiles of four genes using R software and bioinformatics tools. A total of 147 differential genes were identified using RRA analysis, including 73 downregulated and 74 upregulated. GO analysis indicated that the upregulated DEGs were primarily associated with microtubule binding and the downregulated DEGs with serine-type peptidase activity. KEGG pathway analysis indicated that these DEGs were primarily enriched in the cell cycle.

Our findings echo with the previous studies. It has been reported that microtubule binding and cell cycle have an effect on CC[17]. Other studies showed that microtubule binding played a role in the biology of acute myeloid leukemia cells and colorectal cancer cells[18, 19]. Cell cycle also decides the abnormal proliferation of many tumor cells[20, 21]. 
PPI network displayed 30 hub genes associated with CC associated proteins. Next, we found CDK1 was in the center of PPI network of CESC. CDK1 was harbored by top module 1, suggesting that the top module 1 plays a crucial role in CC pathogenesis. Functional analysis indicated that the genes in this module were mainly enriched in microtubule binding, tubulin binding and cell cycle. CDK1 is a serine/threonine kinases that regulates the cell cycle by interacting with specific cyclins[22]. It has been reported that CDK1 regulated the development of CC and many other tumors[23]. Y. Zeng et al. found that Cyclin-dependent kinase 1 (CDK1)-mediated mitotic phosphorylation of the transcriptional co-repressor Vgll4 was mediated by CDK1 to its tumor-suppressing activity. K. Bednarek et al. found that CDK1 was involved in the processes of laryngeal squamous cell carcinoma [24, 25].

Cmap showed that apigenin, thioguanine and trichostatin A could be used to treat CC. Apigenin and trichostatin A can inhibit breast cancer growth[26, 27]. 6-thioguanine has also potential therapeutic effects on tumors[28]. Our findings may help create the appropriate drugs for CC treatment.

Eight genes (APOD, CXCL8, MMP1, MMP3, PLOD2, PTGDS, SNX10 and SPP1) were screened out of DEGs through GEPIA. Of them, only PTGDS and SNX10 had not been reported in CC research. According to GEO validation results, PTGDS was lowly expressed and SNX10 highly expressed in tumor tissues, which was consistent with the results from immunohistochemistry. TCGA methylation analysis showed that the patients with lowly-expressed and highly-methylated PTGDS had a worse prognosis than those with highly-expressed and lowly-methylated PTGDS. Cox regression analysis showed that SNX10 and PTGDS were independent prognostic indicators for OS among CC patients. GSEA showed that the two genes were associated with the chemokine signaling pathway. Zhong $\mathrm{G}$ et al. suggested that chemokine signaling is involved in the invasion and migration of lung cancer cells[29]. Chemokine signaling has also been reported to maneuver the progression of breast and hepatobiliary cancer[30, 31]. In addition, the prognostic signature was constructed based on the eight hub genes. Of them, five genes (SPP1, CXCL8, PTGDS, PLOD2, and MMP3) exhibited significant prognostic value. The Cox regression analysis showed that only the risk score of the five genes was an independent prognostic indicator of CC.

Interestingly, all of the above genes are associated with cervical cancer. Yan $\mathrm{R}$ et al. found that CXCL8 had prognostic value in cervical carcinoma patients[32]. Tian R et al. identified the role of MMP1 in cervical cancer[33]. Xie B et al. defined that genetic polymorphisms in MMP 2, 3, 7, and 9 genes connected with the susceptibility and clinical outcome of cervical cancer in a Chinese Han population [34]. Xu F et al. found that hypoxia and TGF- $\beta 1$ increased PLOD2 expression to facilitate epithelial-to-mesenchymal transition (EMT) and focal adhesion formation, thus promoting the migration and invasion of cervical cancer cells[35]. Chen X et al. found SPP1 inhibition enhanced the chemosensitivity of cervical cancer cell lines to cisplatin[36]. Using microarray analysis, Song JY et al. found that APOD played in the invasion of cervical cancer[37]. These genes play different roles in other tumors. For example, Allina DO et al. demonstrated the diagnostic significance of APOD for prostatic neoplasms[38]. Shen T et al. held that CXCL8 induced EMT in colon cancer [39]. Wang $Y$ et al. found that CXCL8 regulated the development of breast cancer[40]. Ha $\mathrm{H}$ et al. found that CXCL8 was also involved in inflammatory diseases in addition to tumors[41]. Liu M et al. argued that MMP1 promoted the growth and 
metastasis of esophageal squamous cell carcinoma[42]. MMP1 also participated in breast and ovarian cancer[43, 44]. Banik D et al. demonstrated that MMP3 regulated tumor progression[45]. Ji Y et al. demonstrated that C/EBP $\beta$ promoted tumor cell invasion and metastasis of colorectal cancer[46]. PLOD2 is implicated in cervical cancer[35] and renal cell carcinoma[47]. SPP1 linked with lung adenocarcinoma, gastric cancer and colorectal cancer [48-50].

Sorting nexin 10 (SNX10) can suppress the progression of colorectal cancer, liver cancer and stomach cancer[51, 52]. Cervantes-Anaya $\mathrm{N}$ et al. demonstrated SNX10/V-ATPase pathway regulated ciliogenesis in vitro and in vivo[52]. Prostaglandin D synthase (PTGDS), which catalyzes the conversion of prostaglandin $\mathrm{H} 2$ into prostaglandin D2 (PGD2), has been intensely studied [53]. Omori K et al. demonstrated that PTGDS attenuated the malignance of tumor endothelial cells and regulated the processes in gastric cancer and non-small cell lung cancer $[54,55]$. The present study is the first to report the expression and prognostic calue of these two genes in CC. Their methylation is associated with CC prognosis, a finding that has never been reported before.

This study has some limitations. First, the analysis is entirely based on open databases, so its results should be validated with functional experiments. Second, the five genetic profiles are based on a single cohort with relatively small sample size. Future studies should involve larger independent cohorts.

\section{Conclusion}

Our study indicated that two novel genes PTGDS and SNX10 showed abnormal expression and methylation associated with $\mathrm{CC}$ development and explored their prognostic value. However, biofunctions of two genes remained to be unveiled with more in-depth research. The two genes might serve as potential prognostic biomarkers and therapeutic targets in the treatment of CC.

\section{Abbreviations}

Cervical cancer (CC); Differentially expressed gene (DEGs); Gene Expression Omnibus (GEO); RobustRankAggreg冈RRAð; Gene Ontology (GO); Kyoto Encyclopedia of Genes and Genomes (KEGG); Connectivity Map (Cmap); protein-protein interaction (PPI) ; Search Tool for the Retrieval of Interacting Genes Database (STRING) ; Molecular Complex Detection (MCODE); Gene Expression Profiling Interactive Analysis (GEPIA) ; Receiver operating characteristic (ROC) ; Gene set enrichment analysis (GSEA)

\section{Declarations}

\section{Ethics approval and consent to participate}

This study does not involve the use of any animal or human data or tissue. The approval or the consent were not applicable.

\section{Consent to publication}


Not applicable.

\section{Availability of data and material}

The datasets used and/or analysed during the current study are available from the corresponding author on reasonable request.

\section{Competing interests}

The authors declare that they have no competing interests.

\section{Fundings}

This research did not receive any specific grant from funding agencies in the public, commercial, or notfor-profit sectors.

\section{Authors' contributions}

Authors SLF, MXX and PPJ designed the project. Authors YC and FG contributed on data analysis, PPJ, $\mathrm{JHL}$ and WS prepared the main manuscript. ZYM made revisions in language and finalization of the manuscript. All authors have reviewed and approved the manuscript.

\section{Acknowledgements}

Not applicable.

\section{Authors' Information}

${ }^{1}$ Department of Gynecology, The First Affiliated Hospital of Nanjing Medical University, Nanjing 210029, Jiangsu Province, China

${ }^{2}$ Department of Obstetrics and Gynecology, Changzhou Second People's Hospital,Changzhou 213000, Jiangsu, China

${ }^{3}$ Department of Orthopedics, The First Affiliated Hospital of Nanjing Medical University, Nanjing 210029, Jiangsu, China

\section{References}

1. Torre LA, Bray F, Siegel RL, Ferlay J, Lortet-Tieulent J, Jemal A: Global cancer statistics, 2012. CA: a cancer journal for clinicians 2015, 65(2):87-108.

2. Maguire R, Kotronoulas G, Simpson M, Paterson C: A systematic review of the supportive care needs of women living with and beyond cervical cancer. Gynecologic oncology 2015, 136(3):478-490. 
3. Petryszak R, Burdett T, Fiorelli B, Fonseca NA, Gonzalez-Porta M, Hastings E, Huber W, Jupp S, Keays $\mathrm{M}$, Kryvych $\mathrm{N}$ et al: Expression Atlas update-a database of gene and transcript expression from microarray- and sequencing-based functional genomics experiments. Nucleic acids research 2014 , 42(Database issue):D926-932.

4. Nannini M, Pantaleo MA, Maleddu A, Astolfi A, Formica S, Biasco G: Gene expression profiling in colorectal cancer using microarray technologies: results and perspectives. Cancer treatment reviews 2009, 35(3):201-209.

5. Zhai Y, Kuick R, Nan B, Ota I, Weiss SJ, Trimble CL, Fearon ER, Cho KR: Gene expression analysis of preinvasive and invasive cervical squamous cell carcinomas identifies HOXC10 as a key mediator of invasion. Cancer research 2007, 67(21):10163-10172.

6. den Boon JA, Pyeon D, Wang SS, Horswill M, Schiffman M, Sherman M, Zuna RE, Wang Z, Hewitt SM, Pearson $\mathrm{R}$ et al: Molecular transitions from papillomavirus infection to cervical precancer and cancer: Role of stromal estrogen receptor signaling. Proceedings of the National Academy of Sciences of the United States of America 2015, 112(25):E3255-3264.

7. Pappa KI, Polyzos A, Jacob-Hirsch J, Amariglio N, Vlachos GD, Loutradis D, Anagnou NP: Profiling of Discrete Gynecological Cancers Reveals Novel Transcriptional Modules and Common Features Shared by Other Cancer Types and Embryonic Stem Cells. PLoS One 2015, 10(11):e0142229.

8. Ritchie ME, Phipson B, Wu D, Hu Y, Law CW, Shi W, Smyth GK: limma powers differential expression analyses for RNA-sequencing and microarray studies. Nucleic acids research 2015, 43(7):e47.

9. Piwecka M, Rolle K, Belter A, Barciszewska AM, Zywicki M, Michalak M, Nowak S, NaskretBarciszewska MZ, Barciszewski J: Comprehensive analysis of microRNA expression profile in malignant glioma tissues. Molecular oncology 2015, 9(7):1324-1340.

10. Yu G, Wang LG, Han Y, He QY: clusterProfiler: an R package for comparing biological themes among gene clusters. Omics : a journal of integrative biology 2012, 16(5):284-287.

11. von Mering C, Huynen M, Jaeggi D, Schmidt S, Bork P, Snel B: STRING: a database of predicted functional associations between proteins. Nucleic acids research 2003, 31(1):258-261.

12. Bandettini WP, Kellman P, Mancini C, Booker OJ, Vasu S, Leung SW, Wilson JR, Shanbhag SM, Chen MY, Arai AE: MultiContrast Delayed Enhancement (MCODE) improves detection of subendocardial myocardial infarction by late gadolinium enhancement cardiovascular magnetic resonance: a clinical validation study. Journal of cardiovascular magnetic resonance : official journal of the Society for Cardiovascular Magnetic Resonance 2012, 14:83.

13. Lamb J: The Connectivity Map: a new tool for biomedical research. Nature reviews Cancer 2007, 7(1):54-60.

14. Tang Z, Li C, Kang B, Gao G, Li C, Zhang Z: GEPIA: a web server for cancer and normal gene expression profiling and interactive analyses. Nucleic acids research 2017, 45(W1):W98-w102.

15. Rhodes DR, Kalyana-Sundaram S, Mahavisno V, Varambally R, Yu J, Briggs BB, Barrette TR, Anstet MJ, Kincead-Beal C, Kulkarni $P$ et al: Oncomine 3.0: genes, pathways, and networks in a collection of 18,000 cancer gene expression profiles. Neoplasia (New York, NY) 2007, 9(2):166-180. 
16. Subramanian A, Kuehn H, Gould J, Tamayo P, Mesirov JP: GSEA-P: a desktop application for Gene Set Enrichment Analysis. Bioinformatics (Oxford, England) 2007, 23(23):3251-3253.

17. Su Z, Yang H, Zhao M, Wang Y, Deng G, Chen R: MicroRNA-92a Promotes Cell Proliferation in Cervical Cancer via Inhibiting p21 Expression and Promoting Cell Cycle Progression. Oncology research 2017, 25(1):137-145.

18. Pera B, Calvo-Vidal MN, Ambati S, Jordi M, Kahn A, Diaz JF, Fang W, Altmann KH, Cerchietti L, Moore MAS: High affinity and covalent-binding microtubule stabilizing agents show activity in chemotherapy-resistant acute myeloid leukemia cells. Cancer letters 2015, 368(1):97-104.

19. Huda MN, Erdene-Ochir E, Pan CH: Assay for Phosphorylation and Microtubule Binding Along with Localization of Tau Protein in Colorectal Cancer Cells. Journal of visualized experiments : JoVE 2017(128).

20. Roy D, Sheng GY, Herve S, Carvalho E, Mahanty A, Yuan S, Sun L: Interplay between cancer cell cycle and metabolism: Challenges, targets and therapeutic opportunities. Biomedicine \& pharmacotherapy = Biomedecine \& pharmacotherapie 2017, 89:288-296.

21. Newell M, Baker K, Postovit LM, Field CJ: A Critical Review on the Effect of Docosahexaenoic Acid (DHA) on Cancer Cell Cycle Progression. Int J Mol Sci 2017, 18(8).

22. Zhang P, Kawakami H, Liu W, Zeng X, Strebhardt K, Tao K, Huang S, Sinicrope FA: Targeting CDK1 and MEK/ERK Overcomes Apoptotic Resistance in BRAF-Mutant Human Colorectal Cancer. Molecular cancer research : MCR 2018, 16(3):378-389.

23. Luo Y, Wu Y, Peng Y, Liu X, Bie J, Li S: Systematic analysis to identify a key role of CDK1 in mediating gene interaction networks in cervical cancer development. Irish journal of medical science 2016, 185(1):231-239.

24. Zeng Y, Stauffer S, Zhou J, Chen X, Chen Y, Dong J: Cyclin-dependent kinase 1 (CDK1)-mediated mitotic phosphorylation of the transcriptional co-repressor Vgll4 inhibits its tumor-suppressing activity. The Journal of biological chemistry 2017, 292(36):15028-15038.

25. Bednarek K, Kiwerska K, Szaumkessel M, Bodnar M, Kostrzewska-Poczekaj M, Marszalek A, Janiszewska J, Bartochowska A, Jackowska J, Wierzbicka M et al: Recurrent CDK1 overexpression in laryngeal squamous cell carcinoma. Tumour biology : the journal of the International Society for Oncodevelopmental Biology and Medicine 2016, 37(8):11115-11126.

26. Tseng TH, Chien MH, Lin WL, Wen YC, Chow JM, Chen CK, Kuo TC, Lee WJ: Inhibition of MDA-MB231 breast cancer cell proliferation and tumor growth by apigenin through induction of G2/M arrest and histone H3 acetylation-mediated p21(WAF1/CIP1) expression. Environmental toxicology 2017, 32(2):434-444.

27. Chen L, Jin T, Zhu K, Piao Y, Quan T, Quan C, Lin Z: PI3K/mTOR dual inhibitor BEZ235 and histone deacetylase inhibitor Trichostatin A synergistically exert anti-tumor activity in breast cancer. Oncotarget 2017, 8(7):11937-11949.

28. Munshi PN, Lubin M, Bertino JR: 6-thioguanine: a drug with unrealized potential for cancer therapy. The oncologist 2014, 19(7):760-765. 
29. Zhong G, Chen L, Yin R, Qu Y, Bao Y, Xiao Q, Zhang Z, Shen Y, Li C, Xu Y et al: Chemokine (CC motif) ligand 21/CC chemokine receptor type 7 triggers migration and invasion of human lung cancer cells by epithelialmesenchymal transition via the extracellular signalregulated kinase signaling pathway. Mol Med Rep 2017, 15(6):4100-4108.

30. Ehling J, Tacke F: Role of chemokine pathways in hepatobiliary cancer. Cancer letters 2016, 379(2):173-183.

31. Xiao G, Wang X, Wang J, Zu L, Cheng G, Hao M, Sun X, Xue Y, Lu J, Wang J: CXCL16/CXCR6 chemokine signaling mediates breast cancer progression by pERK1/2-dependent mechanisms. Oncotarget 2015, 6(16):14165-14178.

32. Yan R, Shuai H, Luo X, Wang X, Guan B: The clinical and prognostic value of CXCL8 in cervical carcinoma patients: immunohistochemical analysis. Bioscience reports 2017, 37(5).

33. Tian R, Li X, Gao Y, Li Y, Yang P, Wang K: Identification and validation of the role of matrix metalloproteinase-1 in cervical cancer. International journal of oncology 2018, 52(4):1198-1208.

34. Xie B, Zhang Z, Wang H, Chen Z, Wang Y, Liang H, Yang G, Yang X, Zhang H: Genetic polymorphisms in MMP 2, 3, 7, and 9 genes and the susceptibility and clinical outcome of cervical cancer in a Chinese Han population. Tumour biology : the journal of the International Society for Oncodevelopmental Biology and Medicine 2016, 37(4):4883-4888.

35. Xu F, Zhang J, Hu G, Liu L, Liang W: Hypoxia and TGF-beta1 induced PLOD2 expression improve the migration and invasion of cervical cancer cells by promoting epithelial-to-mesenchymal transition (EMT) and focal adhesion formation. Cancer Cell Int 2017, 17:54.

36. Chen X, Xiong D, Ye L, Yang H, Mei S, Wu J, Chen S, Mi R: SPP1 inhibition improves the cisplatin chemo-sensitivity of cervical cancer cell lines. Cancer chemotherapy and pharmacology 2019, 83(4):603-613.

37. Song JY, Lee JK, Lee NW, Jung HH, Kim SH, Lee KW: Microarray analysis of normal cervix, carcinoma in situ, and invasive cervical cancer: identification of candidate genes in pathogenesis of invasion in cervical cancer. International journal of gynecological cancer : official journal of the International Gynecological Cancer Society 2008, 18(5):1051-1059.

38. Allina DO, Andreeva YY, Zavalishina LE, Moskvina LV, Frank GA: [Estimation of the diagnostic potential of APOD, PTOV1, and EPHA4 for prostatic neoplasms]. Arkhiv patologii 2016, 78(5):9-14.

39. Shen T, Yang Z, Cheng X, Xiao Y, Yu K, Cai X, Xia C, Li Y: CXCL8 induces epithelial-mesenchymal transition in colon cancer cells via the PI3K/Akt/NF-kappaB signaling pathway. Oncology reports 2017, 37(4):2095-2100.

40. Wang Y, Liu J, Jiang Q, Deng J, Xu F, Chen X, Cheng F, Zhang Y, Yao Y, Xia Z et al: Human AdiposeDerived Mesenchymal Stem Cell-Secreted CXCL1 and CXCL8 Facilitate Breast Tumor Growth By Promoting Angiogenesis. Stem cells (Dayton, Ohio) 2017, 35(9):2060-2070.

41. Ha H, Debnath B, Neamati N: Role of the CXCL8-CXCR1/2 Axis in Cancer and Inflammatory Diseases. Theranostics 2017, 7(6):1543-1588. 
42. Liu M, Hu Y, Zhang MF, Luo KJ, Xie XY, Wen J, Fu JH, Yang H: MMP1 promotes tumor growth and metastasis in esophageal squamous cell carcinoma. Cancer letters 2016, 377(1):97-104.

43. Yokoi A, Yoshioka Y, Yamamoto Y, Ishikawa M, Ikeda SI, Kato T, Kiyono T, Takeshita F, Kajiyama H, Kikkawa F et al: Malignant extracellular vesicles carrying MMP1 mRNA facilitate peritoneal dissemination in ovarian cancer. Nature communications 2017, 8:14470.

44. Shen CJ, Kuo YL, Chen CC, Chen MJ, Cheng YM: MMP1 expression is activated by Slug and enhances multi-drug resistance (MDR) in breast cancer. PLoS One 2017, 12(3):e0174487.

45. Banik D, Netherby CS, Bogner PN, Abrams SI: MMP3-mediated tumor progression is controlled transcriptionally by a novel IRF8-MMP3 interaction. Oncotarget 2015, 6(17):15164-15179.

46. Ji Y, Li J, Li P, Wang L, Yang H, Jiang G: C/EBPbeta Promotion of MMP3-Dependent Tumor Cell Invasion and Association with Metastasis in Colorectal Cancer. Genetic testing and molecular biomarkers 2018, 22(1):5-10.

47. Kurozumi A, Kato M, Goto Y, Matsushita R, Nishikawa R, Okato A, Fukumoto I, Ichikawa T, Seki N: Regulation of the collagen cross-linking enzymes LOXL2 and PLOD2 by tumor-suppressive microRNA-26a/b in renal cell carcinoma. International journal of oncology 2016, 48(5):1837-1846.

48. Zhang Y, Du W, Chen Z, Xiang C: Upregulation of PD-L1 by SPP1 mediates macrophage polarization and facilitates immune escape in lung adenocarcinoma. Experimental cell research 2017, 359(2):449-457.

49. Xu C, Sun L, Jiang C, Zhou H, Gu L, Liu Y, Xu Q: SPP1, analyzed by bioinformatics methods, promotes the metastasis in colorectal cancer by activating EMT pathway. Biomedicine \& pharmacotherapy = Biomedecine \& pharmacotherapie 2017, 91:1167-1177.

50. Chen LZ, He CY, Su X, Peng JL, Chen DL, Ye Z, Yang DD, Wang ZX, Wang F, Shao JY et al: SPP1 rs4754 and its epistatic interactions with SPARC polymorphisms in gastric cancer susceptibility. Gene 2018, 640:43-50.

51. Zhang J, Wu Y, Jin HY, Guo S, Dong Z, Zheng ZC, Wang Y, Zhao Y: Prognostic value of sorting nexin 10 weak expression in stomach adenocarcinoma revealed by weighted gene co-expression network analysis. World journal of gastroenterology 2018, 24(43):4906-4919.

52. Cervantes-Anaya N, Ponciano-Gomez A, Lopez-Alvarez GS, Gonzalez-Reyes C, Hernandez-Garcia S, Cabanas-Cortes MA, Garrido-Guerrero JE, Villa-Trevino S: Downregulation of sorting nexin 10 is associated with overexpression of miR-30d during liver cancer progression in rats. Tumour biology : the journal of the International Society for Oncodevelopmental Biology and Medicine 2017, 39(4):1010428317695932.

53. Munkholm K, Peijs L, Kessing LV, Vinberg M: Reduced mRNA expression of PTGDS in peripheral blood mononuclear cells of rapid-cycling bipolar disorder patients compared with healthy control subjects. The international journal of neuropsychopharmacology 2014, 18(5).

54. Zhang B, Bie Q, Wu P, Zhang J, You B, Shi H, Qian H, Xu W: PGD2/PTGDR2 Signaling Restricts the Self-Renewal and Tumorigenesis of Gastric Cancer. Stem cells (Dayton, Ohio) 2018, 36(7):990-1003. 
55. He LP, Chen YF, Yang J: [Investigation on the role and mechanism of prostagland in D2 synthase in non-small cell lung cancer]. Zhonghua yi xue za zhi 2017, 97(38):3022-3027.

\section{Tables}

Table 1

Details for GEO cervical cancer data

\begin{tabular}{|llllll|}
\hline Reference & Sample & GEO & Platform & Normal & Tumor \\
\hline Pyeon D,et al(2007) & Cervix & GSE6791 & GPL570 & 8 & 20 \\
\hline Scotto L,et al(2007) & Cervix & GSE9750 & GPL96 & 24 & 33 \\
\hline Espinosa AM,et al(2012) & Cervix & GSE39001 & GPL201 & 12 & 43 \\
& & & GPL6244 & & \\
\hline den Boon JA,et al(2014) & Cervix & GSE63514 & GPL570 & 24 & 28 \\
\hline
\end{tabular}

Table 2

Screening DEGs in cervical cancer by integrated microarray

\begin{tabular}{|ll|}
\hline DEGs & Gene name \\
\hline Upregulated & MMP1 IFI44L MMP12 PLOD2 CXCL11 RFC4 HOXC6 TOP2A ISG15 SPP1 PRC1 \\
& RAD51AP1 SYCP2 DTL APOBEC3B MLF1 TTK CDKN2A INHBA NDC80 EZH2 \\
& CXCL8 KIF23 CTHRC1 MCM2 KIF20A KIF4A CDK1 MICB CENPE LAMP3 IFI44 \\
& CXCL13 CDC25B TOPBP1 CDC7 LMNB1 RRM2 CDC6 HLTF SYNGR3 NCAPG RYR1 \\
& ENO2 SMC4 NEK2 CXCL1 MCM3 C1QB SNX10 PPAP2C KIF11 MCM5 AIM2 AURKA \\
& MAD2L1 PBK CENPF KIF15 KNTC1 NTS FBX05 STIL SPAG5 TRIP13 EPCAM MELK \\
& MMP3 KIF14 GZMB CDC20 CEP55 BUB1B NEFH \\
Downregulated & CRNN MAL CRISP3 CRCT1 SPINK5 ALOX12 KRT13 SPRR3 PPP1R3C KRT1 \\
& SPRR1B APOD SPR1A CFD IVL CXCL14 RHCG SPRR2B ENDOU EDN3 CRYAB \\
& TMPRSS11D CLIC3 HPGD UPK1A TST KLK11 BBOX1 EMP1 CLCA4 KLK12 \\
& SCNN1B NSG1 SLURP1 SOSTDC1 IL1R2 KRT4 KLF4 DSG1 PPL DEFB1 SULT2B1 \\
& GPX3 TGM3 ALOX12B ECM1 NDN ILL1 CRABP2 FCGBP PTGDS TMPRSS11B \\
& CCND1 FOSB GYS2 TGFBR3 LDOC1 S100A7 KRT2 FGFBP1 PRSS3 ID4 ADRB2 \\
& VAT1 SLIT2 CLDN8 KLK10 PTK6 SPINK2 AR PDGFD AKR1B10 EREG
\end{tabular}


Table 3

Results of CMap analysis

\begin{tabular}{|llllll|}
\hline Rank & CMap name & Mean & $\mathbf{N}$ & Enrichment & P-value \\
\hline 1 & apigenin & -0.848 & 4 & -0.973 & 0 \\
\hline 2 & thioguanosine & -0.799 & 4 & -0.96 & 0 \\
\hline 3 & trichostatin A & -0.386 & 182 & -0.261 & 0 \\
\hline 4 & viomycin & 0.751 & 4 & 0.924 & 0.00004 \\
\hline 5 & adiphenine & 0.779 & 5 & 0.907 & 0.00004 \\
\hline 6 & atractyloside & 0.651 & 5 & 0.839 & 0.00024 \\
\hline 7 & chrysin & -0.745 & 3 & -0.939 & 0.00032 \\
\hline 8 & isoflupredone & 0.768 & 3 & 0.937 & 0.00044 \\
\hline 9 & nadolol & 0.649 & 4 & 0.866 & 0.00044 \\
\hline
\end{tabular}


Table 4. Univariate analysis and multivariate analysis of SNX10 and PTGDS expression among cervical cancer patients.

\begin{tabular}{|c|c|c|c|c|c|c|}
\hline \multirow[t]{2}{*}{ Variables } & \multicolumn{3}{|c|}{ Univariate analysis } & \multicolumn{3}{|c|}{ Multivariate analysis } \\
\hline & HR & $95 \% \mathrm{CI}$ & $p$ & HR & $95 \% \mathrm{CI}$ & $p$ \\
\hline $\begin{array}{l}\text { Stage } \square \text { I \& } \\
\text { II } \square \text { vs }\end{array}$ & 2.338 & $\begin{array}{l}1.364- \\
4.004\end{array}$ & 0.002 & 3.078 & $\begin{array}{l}1.329- \\
7.129\end{array}$ & 0.009 \\
\hline \multicolumn{7}{|l|}{$\begin{array}{l}\text { Stage } \square \text { III \& } \\
\text { IV } \square\end{array}$} \\
\hline $\begin{array}{l}\text { Grade(G1 } \\
\& \text { G2』vs }\end{array}$ & 1.221 & $\begin{array}{l}0.947- \\
1.574\end{array}$ & 0.124 & 0.837 & $\begin{array}{l}0.565- \\
1.240\end{array}$ & 0.375 \\
\hline \multicolumn{7}{|l|}{ Grade $\llbracket G 3)$} \\
\hline $\begin{array}{l}\text { Age } \square \leq 50 \square \text { vs } \\
\text { Age } \square>50)\end{array}$ & 1.263 & $\begin{array}{l}0.755- \\
2.112\end{array}$ & 0.373 & 1.197 & $\begin{array}{l}0.711- \\
2.011\end{array}$ & 0.498 \\
\hline $\begin{array}{l}\text { SNX10 } \\
\text { (high } \\
\text { expression) } \\
\text { vs }\end{array}$ & 1.202 & $\begin{array}{l}0.957- \\
1.509\end{array}$ & 0.113 & 1.424 & $\begin{array}{l}1.103- \\
1.838\end{array}$ & 0.007 \\
\hline \multicolumn{7}{|l|}{$\begin{array}{l}\text { SNX10 (low } \\
\text { expression) }\end{array}$} \\
\hline $\begin{array}{l}\text { PTGDS } \\
\text { (high } \\
\text { expression) } \\
\text { vs }\end{array}$ & 0.838 & $\begin{array}{l}0.729- \\
0.962\end{array}$ & 0.012 & 0.802 & $\begin{array}{l}0.693- \\
0.928\end{array}$ & 0.003 \\
\hline $\begin{array}{l}\text { PTGDS } \\
\text { (low } \\
\text { expression) }\end{array}$ & & & & & & \\
\hline
\end{tabular}

\section{Supplementary Information}

Figure S1. Standardization of gene expression. The blue bar represents the data before normalization, and the red bar represents the normalized data.(A) The standardization of GSE6791 data, (B) the standardization of GSE9750 data, (C) the standardization of GSE39001 data (D) the standardization of GSE63514 data.

Figure S2. Differential expression of data between two sets of samples. The red points represent upregulated genes screened on the basis of Ifold change| .2.0 and a corrected P-value of ,0.05. The green 
points represent downregulation of the expression of genes screened on the basis of |fold change| .2.0 and a corrected P-value of, 0.05 . The black points represent genes with no significant difference. FC is the fold change.(A)GSE6791 data, (B) GSE9750 data, (C) GSE39001 data and (D) GSE63514 data.

Figure S3. Hierarchical clustering heatmap of DEGs screened on the basis of |fold changel .2.0 and a corrected P-value, 0.05 . Red indicates that the expression of genes is relatively upregulated, green indicates that the expression of genes is relatively downregulated, and black indicates no significant changes in gene expression; gray indicates that the signal strength of genes was not high enough to be detected. (A) GSE6791 data, (B) GSE9750 data, (C) GSE39001 data and (D) GSE63514 data.

Figure S4. GO enrichment analysis of DEGs in cervical cancer. (A) GO analysis divided DEGs into three functional groups: molecular function, biological processes, and cell composition. (B) GO enrichment significance items of DEGs in different functional groups.(C) Distribution of DEGs in cervical cancer for different GO-enriched functions.

Figure S5. (A) Significant function enrichment of DEGs. Blue represents the GO groups, green represents downregulated genes and red represents upregulated genes. (B) Significant pathway enrichment of DEGs. Blue represents signaling pathway, green represents downregulated genes and red represents upregulated genes.

Figure S6. (A) A significant module selected from protein-protein interaction network. (B)GO analysis of these significant molecule.(C) KEGG analysis of these significant molecule.

Figure S7. Validation of genes expression in GEPIA. (A) APOD, (B) CXCL8, (C) MMP1, (D) MMP3, (E) PLOD2, (F) PTGDS, (G) SNX10, (H) SPP1.

Figure S8. Survival analysis of genes in GEPIA. (A) APOD, (B) CXCL8, (C) MMP1, (D) MMP3, (E) PLOD2, (F) PTGDS, (G) SNX10, (H) SPP1.

Figure S9. Gene expression in GSE7803 and GSE29570. (A) PTGDS in GSE7803. (B) SNX10 in GSE7803. (C) PTGDS in GSE29570. (D) SNX10 in GSE29570.

Figure S10. Seven genes were screened by Univariate cox regression analysis.

Figure S11. Five genes were screened by Multivariate cox regression analysis

Figure S12. Regression analysis of the 5 genes identified. (A) The expression level of the 5 genes in lowand high-risk groups. (B) The univariate Cox proportional hazards regression. (C) The multivariate Cox proportional hazards regression. (D) The heatmap of the 5 genes in high- and low-risk patients in TCGA dataset.

\section{Figures}




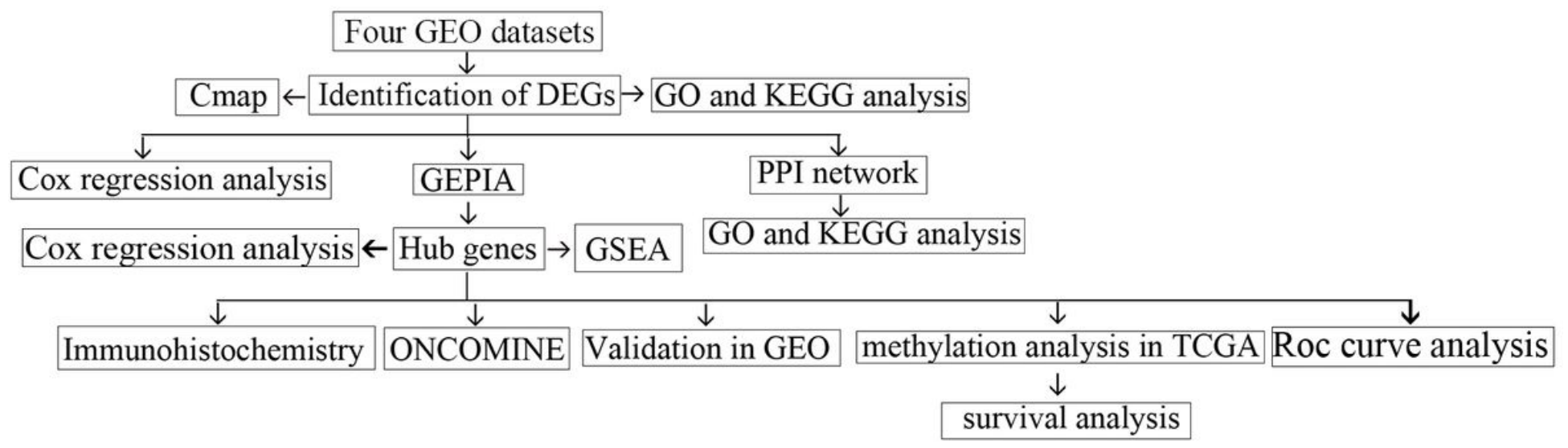

\section{Figure 1}

Flow chart of the present study. 


\begin{tabular}{|c|c|c|c|c|}
\hline 5.74 & 2.52 & 2.66 & 3.90 & MMP1 \\
\hline 3.35 & 1.80 & 4.17 & 2.77 & IFI44L \\
\hline 2.84 & 3.30 & 5.00 & 2.47 & MMP12 \\
\hline 2.10 & 2.11 & 2.28 & 2.70 & PLOD2 \\
\hline 1.98 & 1.53 & 2.91 & 2.22 & CXCL11 \\
\hline 1.93 & 2.75 & 2.96 & 2.39 & RFC4 \\
\hline 2.49 & 1.63 & 3.96 & 2.16 & HOXC6 \\
\hline 1.97 & 2.58 & 2.13 & 2.21 & TOP2A \\
\hline 2.74 & 1.78 & 2.13 & 2.91 & ISG15 \\
\hline 4.26 & 1.96 & 2.17 & 2.13 & SPP1 \\
\hline 1.81 & 2.88 & 2.06 & 2.20 & PRC1 \\
\hline 1.76 & 1.60 & 2.19 & 3.09 & RAD51AP1 \\
\hline 3.60 & 2.57 & 2.64 & 2.09 & SYCP2 \\
\hline 2.78 & 0.00 & 3.32 & 2.96 & DTL \\
\hline 1.65 & 2.35 & 2.37 & 2.75 & APOBEC3B \\
\hline 1.64 & 1.25 & 3.49 & 2.18 & MLF1 \\
\hline 1.64 & 1.86 & 2.11 & 2.00 & TTK \\
\hline 3.69 & 2.67 & 2.94 & 1.95 & CDKN2A \\
\hline 2.92 & 1.14 & 2.64 & 1.95 & INHBA \\
\hline 1.58 & 1.85 & 2.31 & 3.35 & NDC80 \\
\hline-7.43 & -3.33 & -9.39 & -4.52 & CRNN \\
\hline-5.79 & -3.15 & -6.31 & -4.35 & MAL \\
\hline-6.68 & -3.17 & -8.83 & -3.58 & CRISP3 \\
\hline-5.07 & -2.97 & -5.38 & -3.67 & CRCT1 \\
\hline-4.43 & -2.76 & -5.85 & -3.22 & SPINK5 \\
\hline-5.15 & -2.46 & -5.20 & -3.65 & ALOX12 \\
\hline-3.98 & -2.59 & -4.63 & -3.30 & KRT13 \\
\hline-4.18 & -2.46 & -4.42 & -2.02 & SPRR3 \\
\hline-4.16 & -2.48 & -3.54 & -1.79 & PPP1R3C \\
\hline-5.93 & -1.82 & -5.22 & -5.44 & KRT1 \\
\hline-3.09 & -1.96 & -3.38 & -1.86 & SPRR1B \\
\hline-2.33 & -3.80 & -3.53 & -1.85 & APOD \\
\hline-3.77 & -1.65 & -3.66 & -1.72 & SPRR1A \\
\hline-2.62 & -4.01 & -3.11 & -2.39 & CFD \\
\hline-3.78 & -1.51 & -4.34 & -2.61 & IVL \\
\hline-1.94 & -3.24 & -3.35 & -2.62 & CXCL14 \\
\hline-5.08 & -1.45 & -4.65 & -2.57 & RHCG \\
\hline-4.66 & -1.49 & -2.78 & -1.70 & SPRR2B \\
\hline-5.73 & -1.06 & -5.83 & -3.07 & ENDOU \\
\hline-4.11 & -2.38 & -2.73 & -1.75 & EDN3 \\
\hline 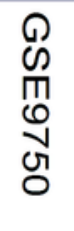 & 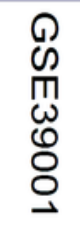 & 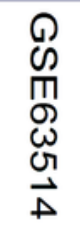 & 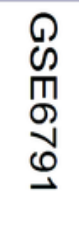 & \\
\hline
\end{tabular}

Figure 2

LogFC heatmap of the image data of each expression microarray. Notes: The abscissa is the geo ID, and the ordinate is the gene name. red represents $\log \mathrm{FC} \otimes 0$, green represents $\log \mathrm{FC} \otimes 0$, and the values in the box represent the logFC values. 

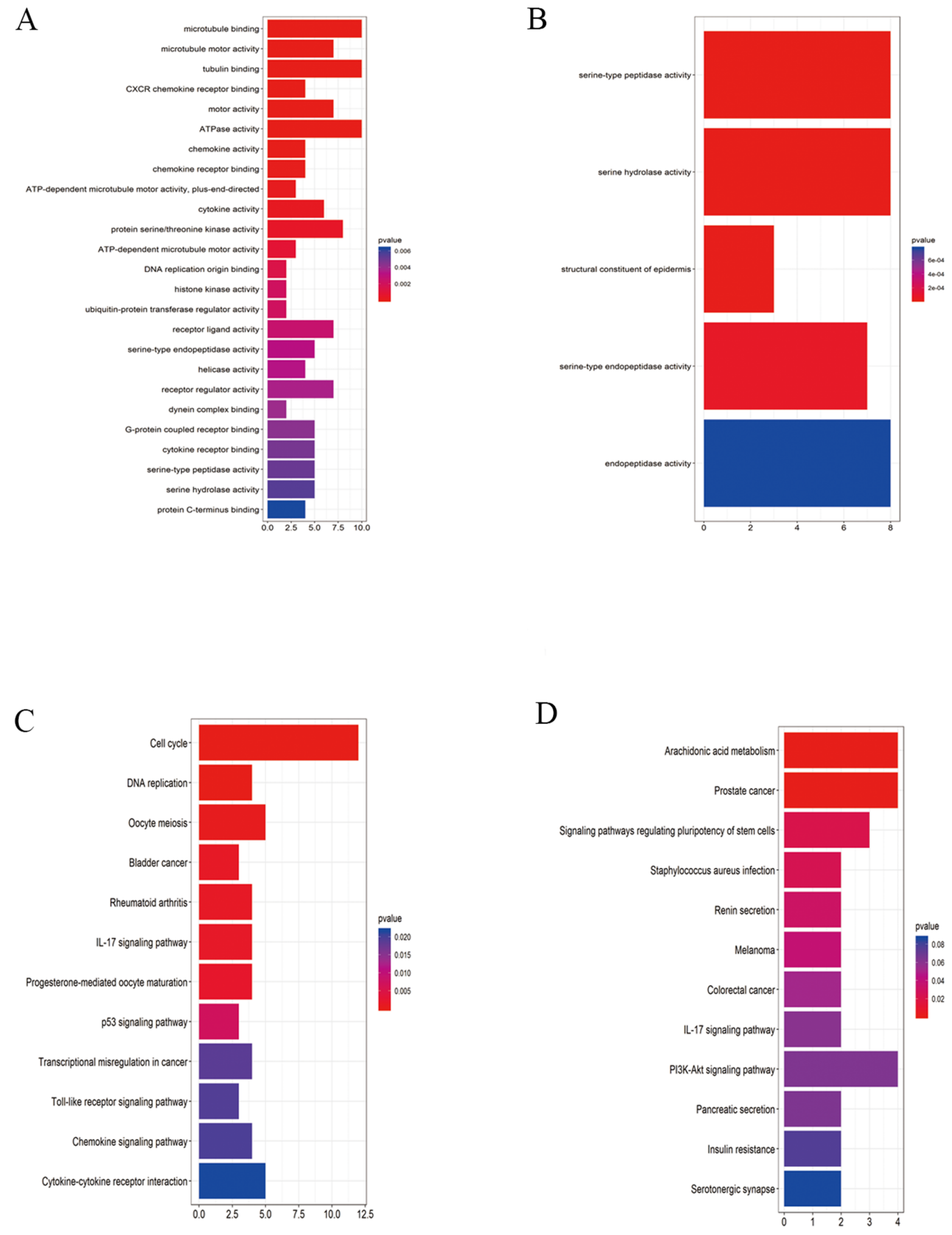

\section{Figure 3}

GO and KEGG analysis of the DEGs. (A) GO analysis of upregulated genes associated with cervical cancer. (B) GO analysis of downregulated genes associated with cervical cancer. (C) KEGG analysis of upregulated genes associated with cervical cancer. (D) KEGG analysis of downregulated genes associated with cervical cancer. 
A

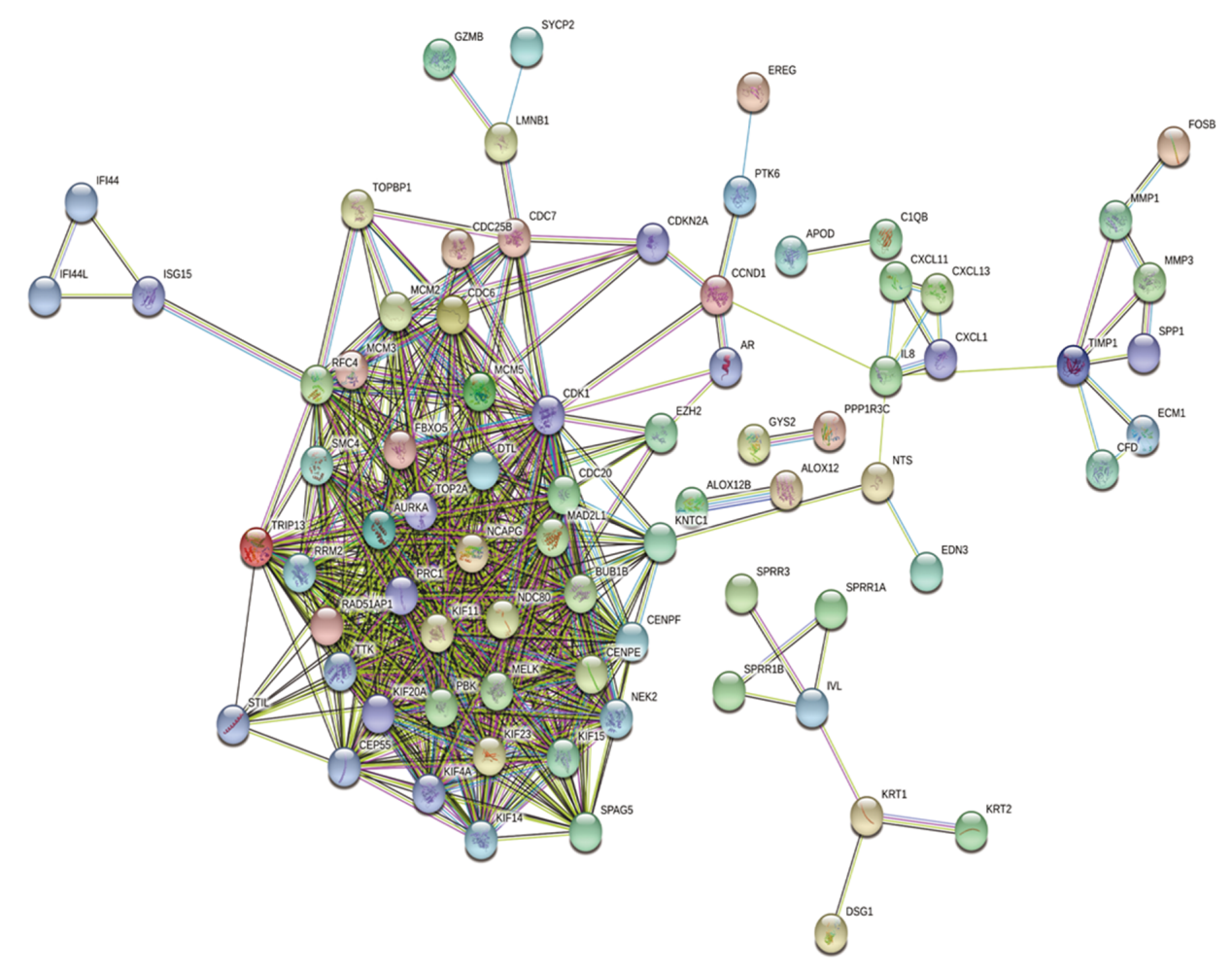

B

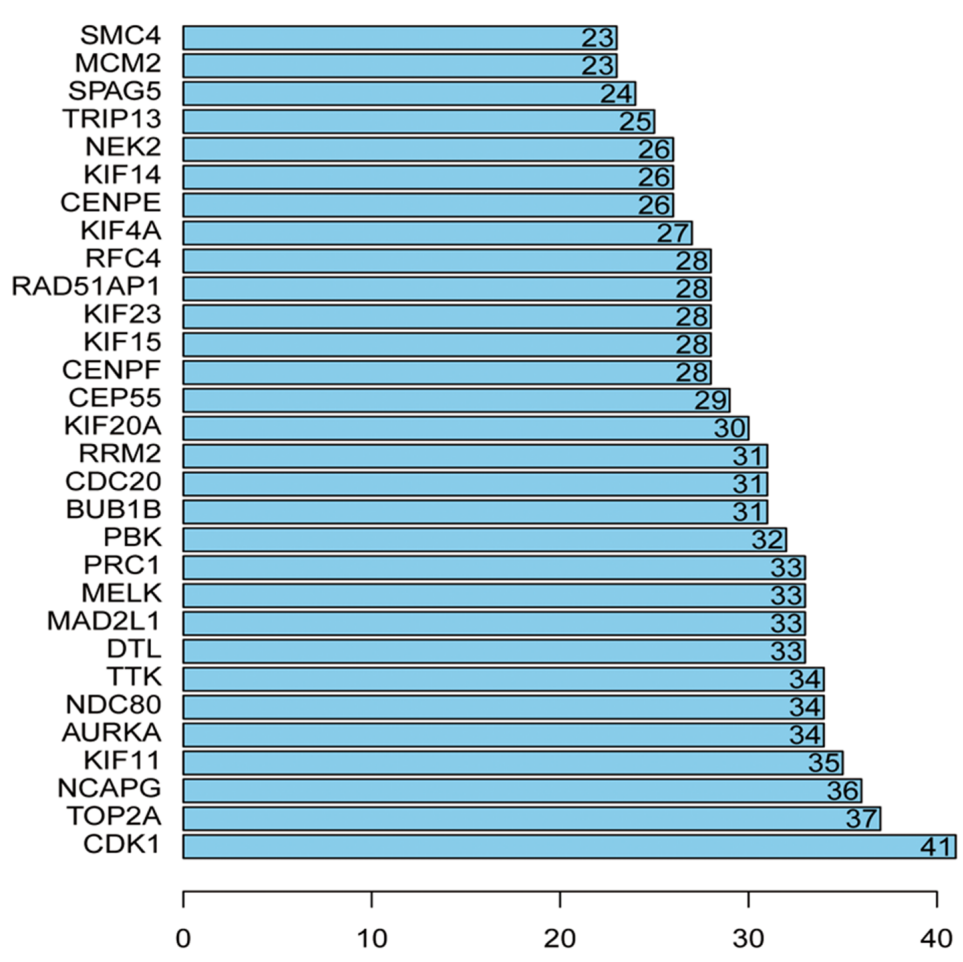

Figure 4

PPI network analysis. (A) Using the STRING online database, a total of 147 DEGs were filtered into the DEGs PPI network. (B) Degree of the top 30 nodes in the PPI network. All these nodes are upregulated genes. 
A

B

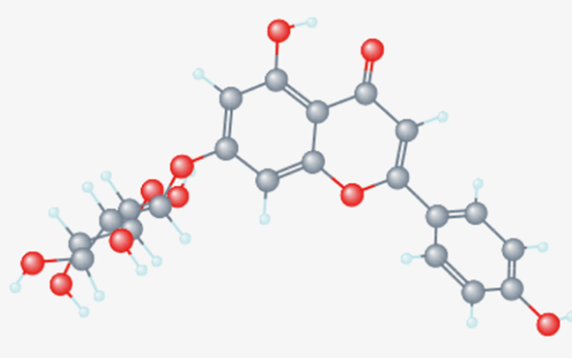

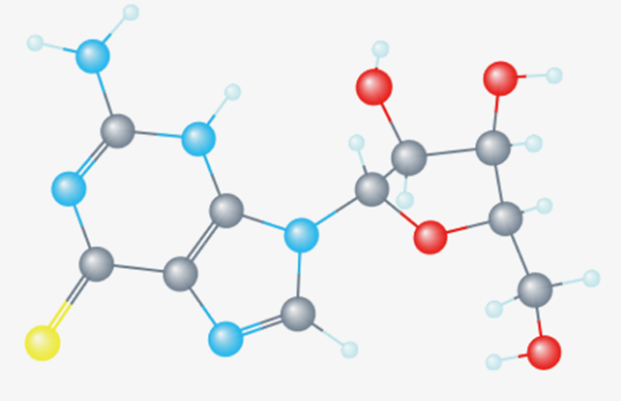

$\mathrm{C}$

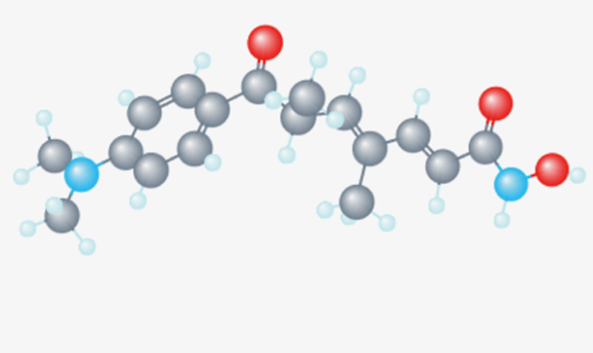

\section{Figure 5}

Three-dimensional diagram of the three most significant drugs. (A)Apigenin (B) Thioguanosine (C) Trichostatin A

\section{PTGDS}

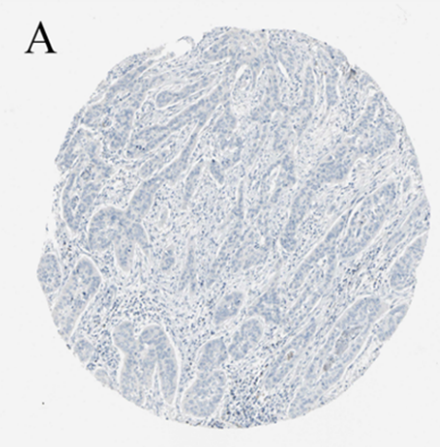

Cancer

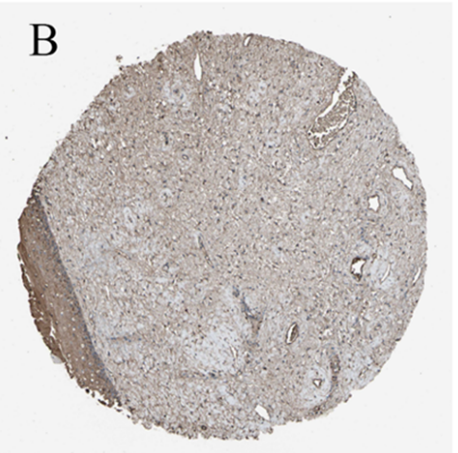

Normal

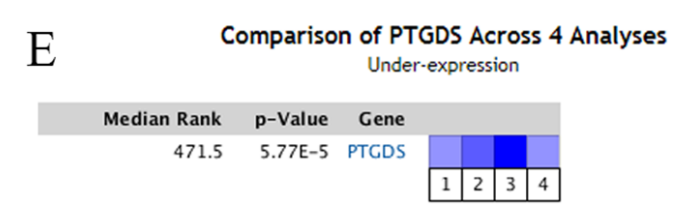

Legend

1. Cervical Squamous Cell Carcinoma vs. 3. Cenvical Cancer Type Epithelia: Cenvical

Normal Squamous Cell Carcinoma

2. Cervical Squamous Cell Carcinoma vs. 4. Cervical Squamous Cell Carcinoma

Normal

Scotto Cervix 2, Genes Chromosomes Zhai Cervix, Cancer Res, 2007 Cancer, 2008

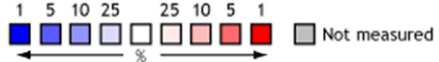

The rank for a gene is the median rank for that gene across each of the analyses.
The $\mathrm{p}$-Value for a gene is its $\mathrm{p}$-Value for the median-ranked analysis.
SNX10

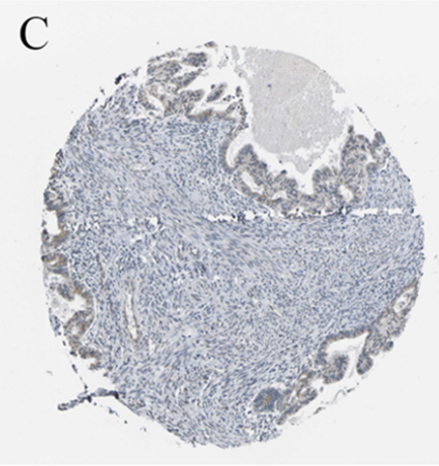

Cancer

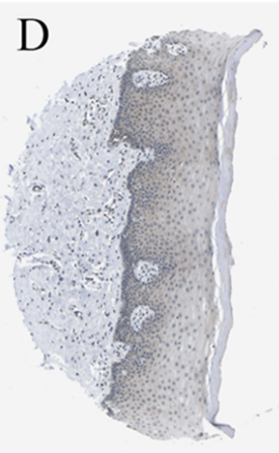

Normal

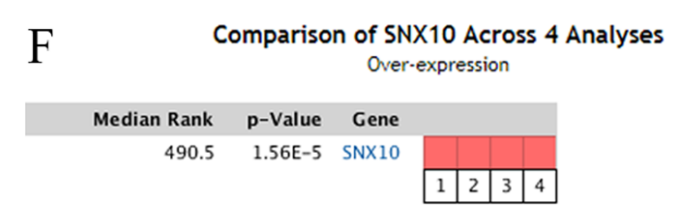

Legend

1. Cervical Squamous Cell Carcinoma vs. 3. Cervical Squamous Cell Carcinoma vs.

$\begin{array}{ll}\text { Normal } & \text { Normal } \\ \text { Biewenga Cervix, Gynecol Oncol, } 2008 & \text { Scotto Cervix 2, Genes Chromosomes }\end{array}$

2. Cenvical Cancer vs. Normal Cancer, 2008

Pyeon Multi-cancer, Cancer Res, 2007 4. Cenvical Squamous Cell Carcinoma Epithelia vs. Normal

Zhai Cervix, Cancer Res, 2007

$\stackrel{1}{5} \square^{10} \square^{25} \square \square_{\%}^{25} \square^{10} \square^{1} \square$ Not measured

The rank for a gene is the median rank for that gene across each of the analyses.
The $\mathrm{p}$-Value for a gene is its $\mathrm{p}$-Value for the median-ranked analysis.

\section{Figure 6}

Validation of PTGDS and SNX10. (A) Immunohistochemistry of PTGDS. (B) Immunohistochemistry of SNX10. (C) Expression of PTGDS in ONCOMINE. (D) Expression of SNX10 in ONCOMINE. 
A

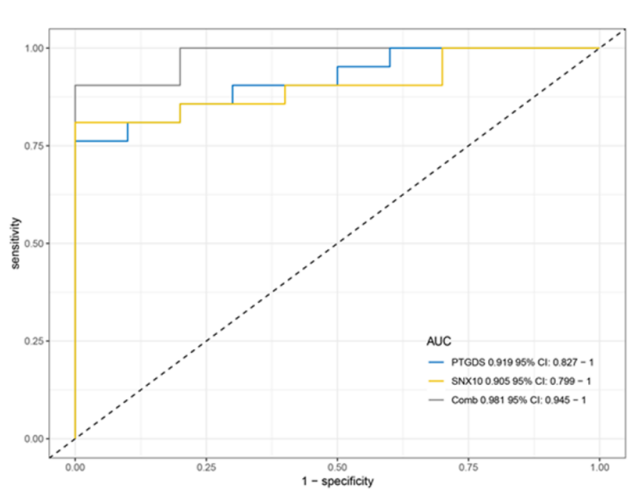

$\mathrm{C}$

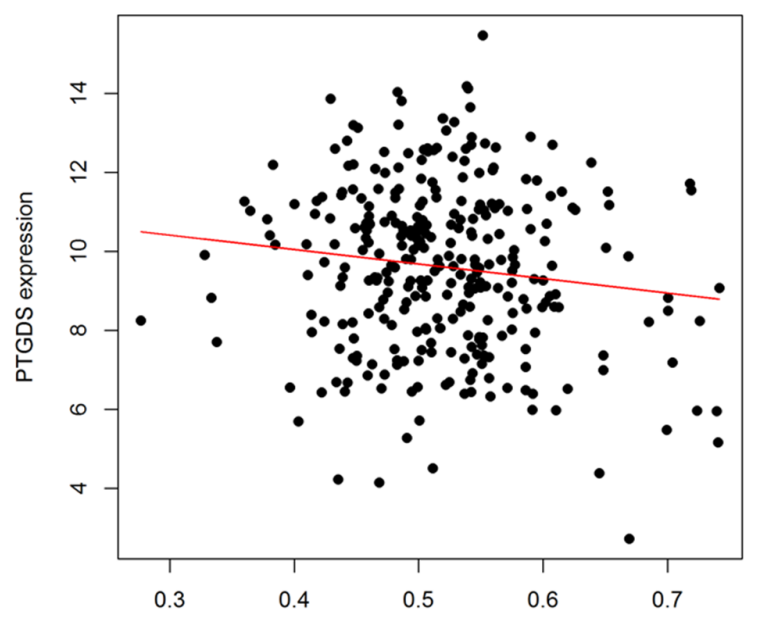

$\mathrm{E}$

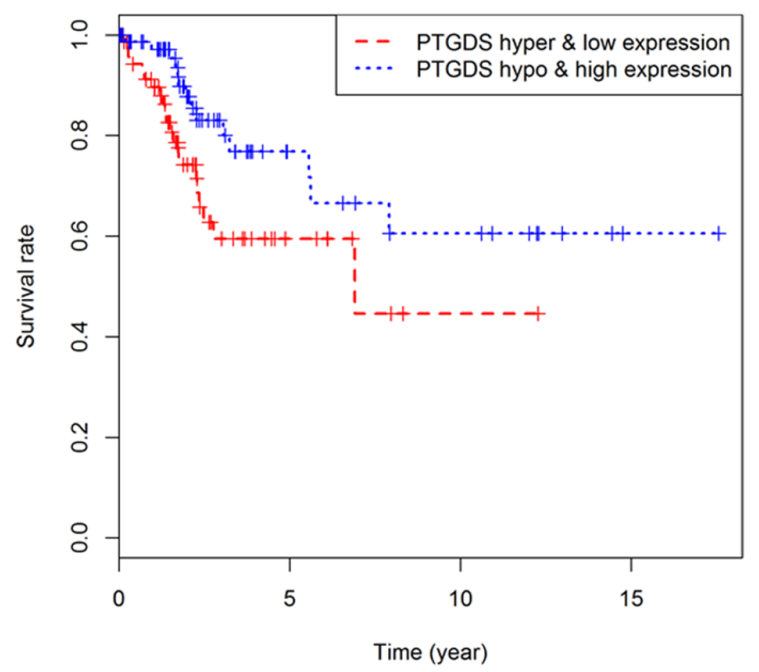

$\mathrm{B}$

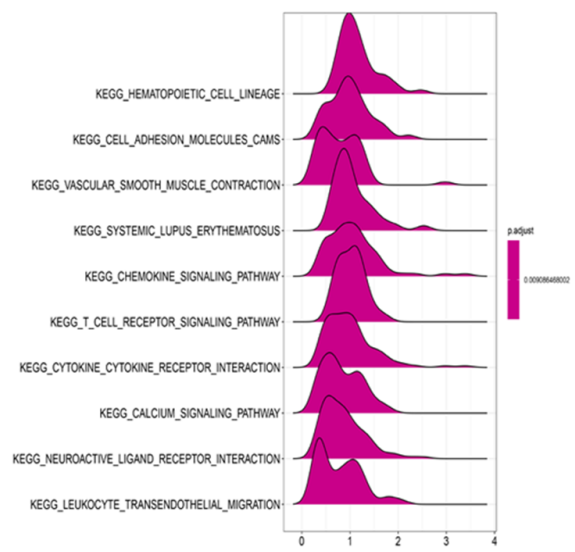

$\mathrm{D}$

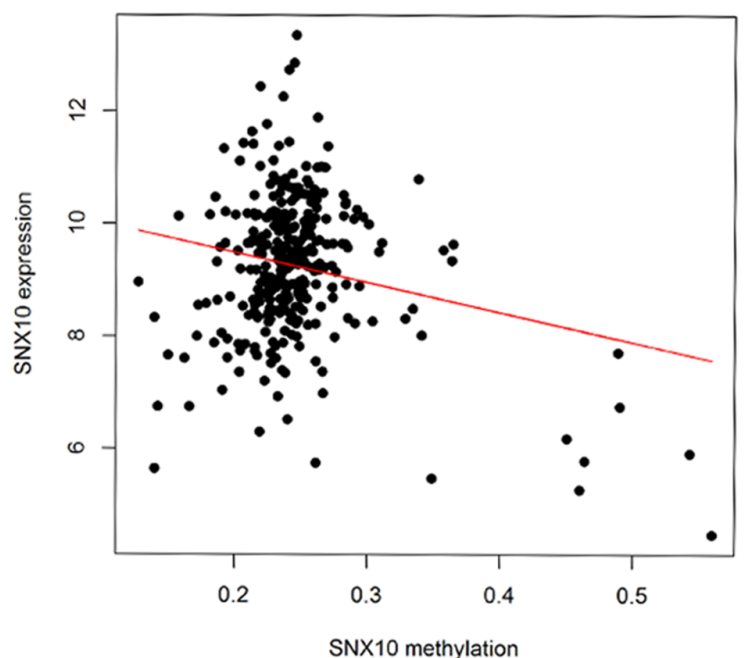

\section{Figure 7}

Validation of PTGDS and SNX10. (A) ROC curve analysis of the two genes. (B) GSEA of PTGDS and SNX10. (C) The methylated expression and gene expression of PTGDS. (D) The methylated expression and gene expression of SNX10. (E) Survival analysis of patients with methylated PTGDS expression. 
A

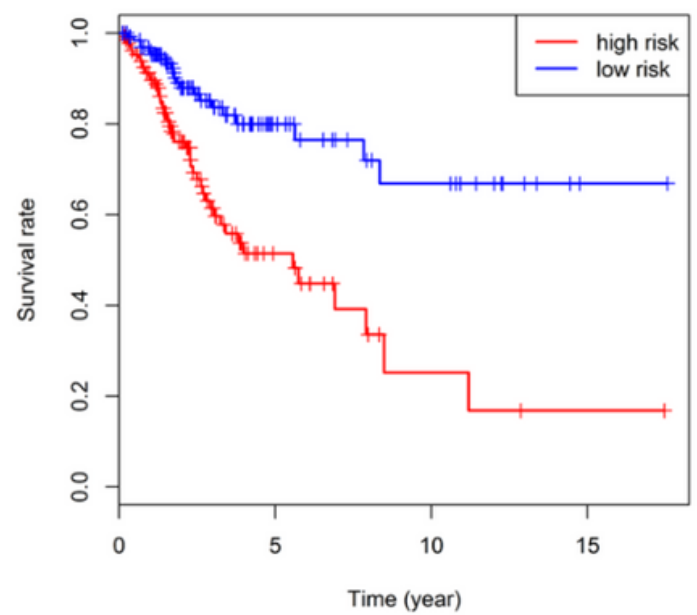

B

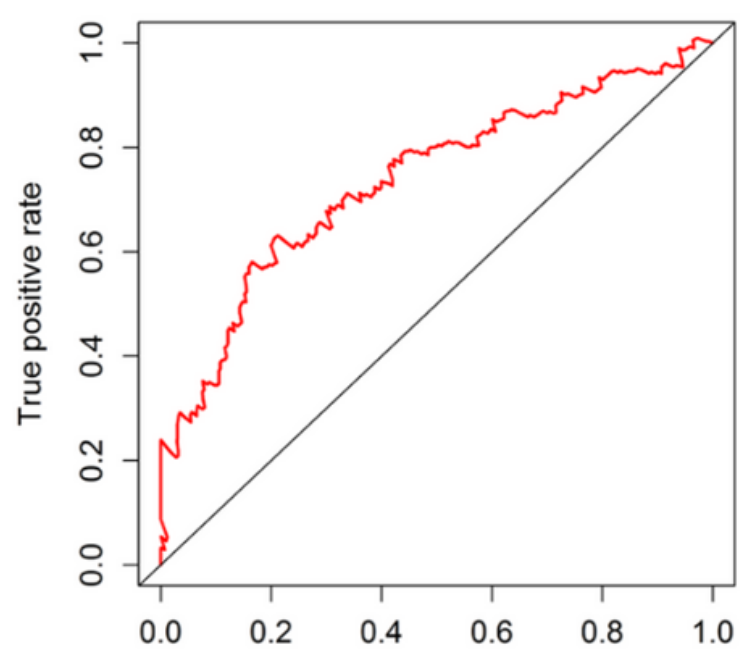

False positive rate
$\mathrm{C}$

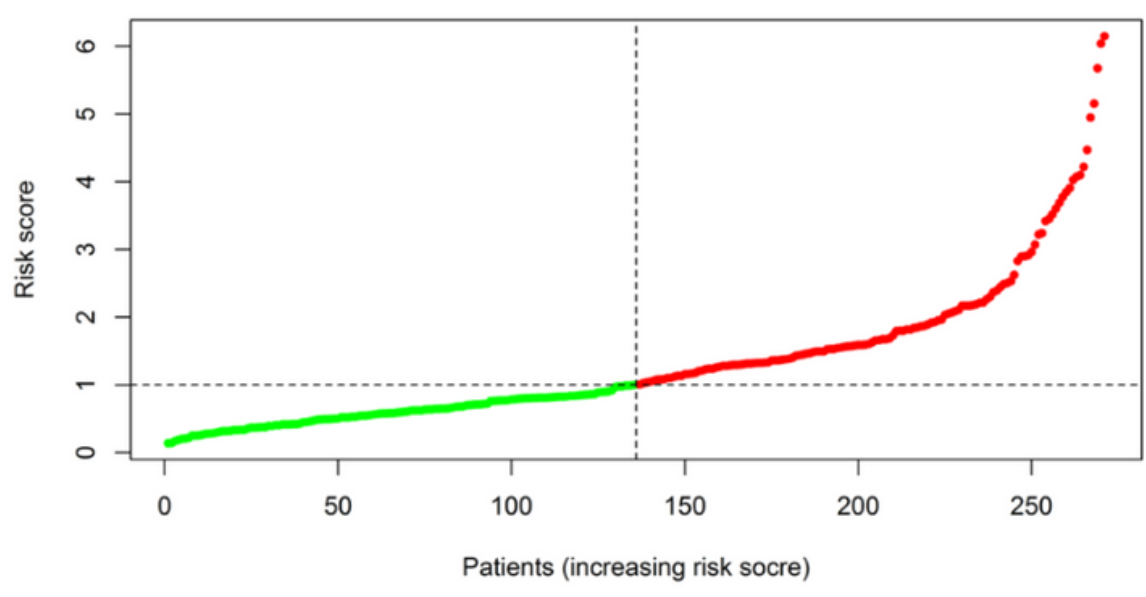

$\mathrm{D}$

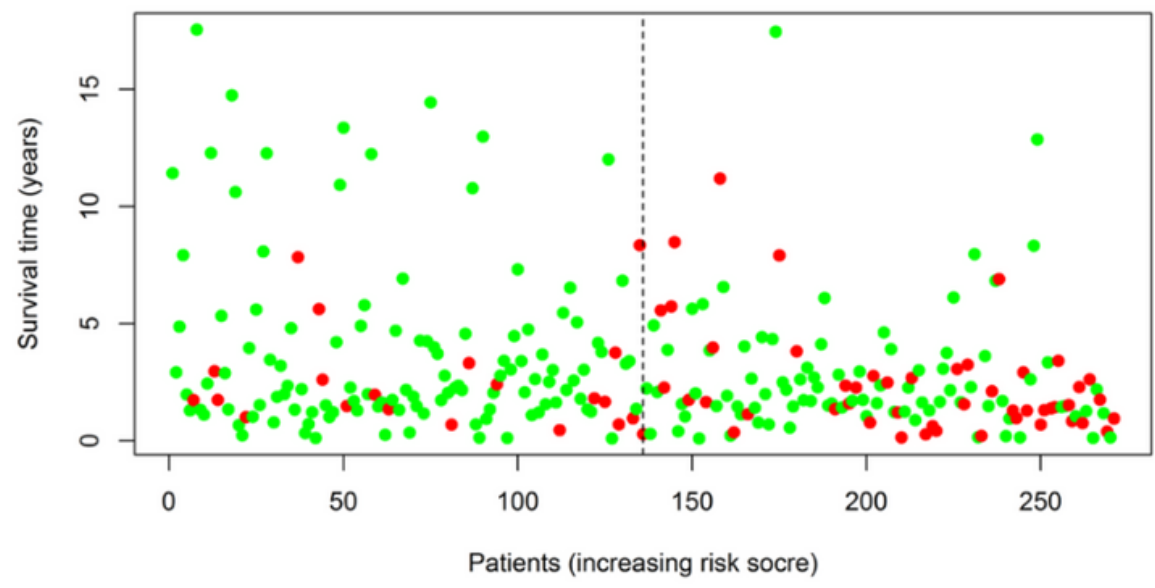

E

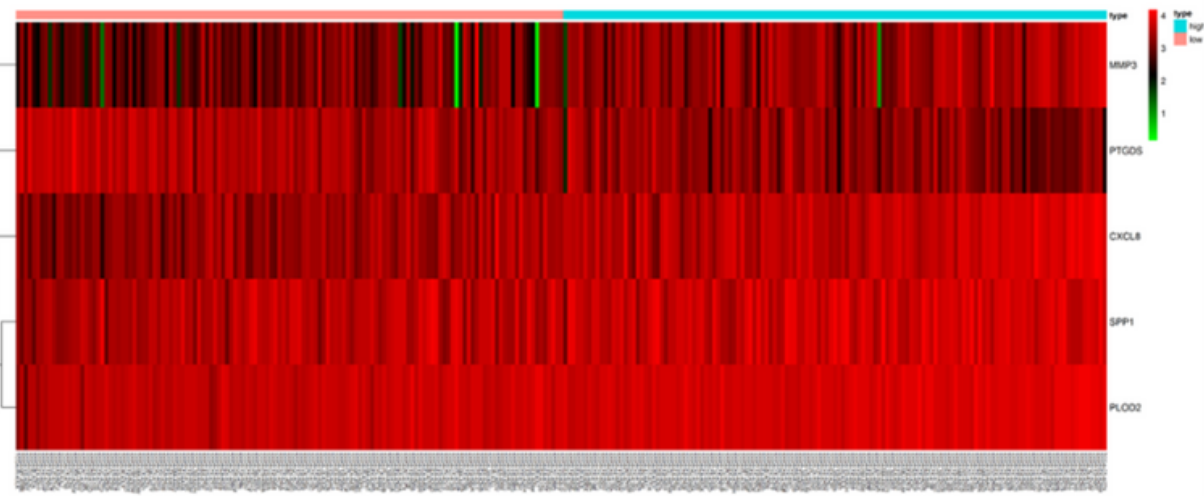

Figure 8

Survival prognosis model of the 5 hub genes. (A) Survival analysis showed that the patients in the high risk group had statistically significantly worse overall survival than those in low risk group in TCGA cohort. (B) ROC analysis was performed to find out the most optimal cutoff value to divide the CC patients into high risk and low risk group. (C-D) The risk scores for all patients in TCGA cohort are plotted in ascending order and marked as low risk (blue) or high risk (red), as divided by the threshold (vertical 
black line). (E) Eight expression and risk score distribution in TCGA cohort by z-score, with red indicating higher expression and light blue indicating lower expression.

\section{Supplementary Files}

This is a list of supplementary files associated with this preprint. Click to download.

- FigureSupp.docx

- SupplementaryFigure12.tif

- SupplementaryFigure11.tiff

- SupplementaryFigure10.tif

- SupplementaryFigure9.tif

- SupplementaryFigure8.tif

- SupplementaryFigure7.tif

- SupplementaryFigure6.tif

- SupplementaryFigure5.tif

- SupplementaryFigure4.tif

- SupplementaryFigure3.tif

- SupplementaryFigure2.tif

- SupplementaryFigure1.tif 